\title{
Study of Impact of Computer Vision in Detecting Human Emotions
}

\author{
Ravindra Kumar
}

\begin{abstract}
Emotions play a powerful role in people's thinking and behaviors. Emotions act as a compulsion to take any action and can influence daily life decisions. Human facial expressions show humans share the same set of emotions. From the setting, the concept of emotion-sensing facial recognition was brought up. Humans have been working actively on computer vision algorithms, the algorithm will help determine the emotions of an individual and can determine the set of intentions accompanied by the emotions. The emotion-sensing facial expression computers are designed using data-centric skills in machine learning and can achieve their desired work by emotion identification and a set of intentions related to the emotion obtained.
\end{abstract}

Keyword: From the setting, the concept of emotion-sensing facial recognition was brought up.

\section{INTRODUCTION}

This paper consists of the study in computer recognition of emotions based on the individual's facial expressions. The classifier for spontaneous emotion detection is based on SVM (support vector machine). The facial representation is created through a face detection algorithm. To use as a facial recognition mechanism, face texture is encoded with local recognition mechanism. The classifier is considered useful and is widely used for aggression classification based upon gestures and auto emotion exploration.

\section{INTRODUCTION OF EMOTION-SENSING FACIAL RECOGNITION}

A large percentage of human communications is through facial expressions (Mehta, 2018). Emotion recognition was not considered at the early stages of its introduction because it was associated with errors in codes. However, in the modern world emotion detection in humans through computer vision is widespread. Gaining an understanding of the contexts of emotions has a set of impacts on society and businesses. Emotion sensing facial recognition can be used by the national government in determinations of emotions to get the intent, the setting can be used to show the emotion of guilt, fear, and uncertainty in an individual's emotions. Corporations and companies have also used computer vision in detecting human emotions to drive their company

Manuscript received on July 29, 2021.

Revised Manuscript received on September 21, 2021.

Manuscript published on September 30, 2021.

* Correspondence Author

Ravindra Kumar*, Technical Account Manager, Navvis Healthcare, St. Louis MO, 63021. Email: rakumar25@gmail.com

(c) The Authors. Published by Blue Eyes Intelligence Engineering and Sciences Publication (BEIESP). This is an open access article under the CC BY-NC-ND license (http://creativecommons.org/licenses/by-nc-nd/4.0/) binary patterns and the feature is used as an emotion

outcomes. Computer vision in detecting human emotions requires the application of two techniques; computer vision is used in the determination of the facial expressions of an individual or the individual whose facial expressions are being analyzed (Anusha, 2020). Application of machine learning algorithm, the algorithm is used in the evaluation of the emotions and the facial features. The algorithm applies supervised learning to recognize things that seem to have happened before. The identification of the emotion is labeled according to the features in the image. For example, it labels an image sad whenever it identifies a sad face. However, it is divided into three stages, face detection is the first stage purposed to at pre-processing of the images. The face is identifying and its unique features are captured in the region (Dzedzickis, Human emotion recognition: Review of sensors and methods, 2020). The indicated in the facial detection are ayes, brows, nose, and mouth. Feature extraction is the second stage in computer visual detection, this is a region where the informative features of the face are extracted from its different parts. Emotion classification is the last stage of emotion visual detection, at this stage, the classifier is trained using the training data before generating the labels. Emotion detection can be applied in various fields, one of the applications is in the evaluation of job applicants, and this application helps companies to evaluate the state of mind and the integrity of the various employees. Such artificial intelligence is applied by corporations for entry-level job evaluation. An example of a corporation is Unilever Company. After an online job application, the interviewers are administered a video interview. At this stage, the artificial intelligence enters their pictures into the system where their moods and state of mind are evaluated. Artificial intelligence has enabled many corporations to employ desired employees who are of good integrity (McStay, 2020). The AI is also applied in criminal evaluation for any signs of deception (Amjad, 2020). The criminal justice system is adopting artificial intelligence for various alleged's evaluations.

\section{ETHICAL DILEMMAS POSED BY THE INTRODUCTION OF EMOTIONAL SENSING FACIAL RECOGNITION}

\section{A. Privacy Invasion}

Invasion of privacy was a major issue raised by individuals. The camera or computer vision users do not know if the computers sending their pictures to be analyzed are also filming them (Koshimizu, 2006). When the facial expressions are taken from different angles are unprecedented in stemming from emotional surveillance.

Published By:

Blue Eyes Intelligence Engineering

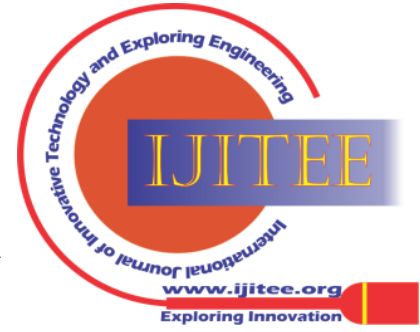




\section{Study of Impact of Computer Vision in Detecting Human Emotions}

Artificial intelligence has introduced new methods to human manipulation by using political propaganda as the targets or the retrieval of passwords through the detection of the micro reaction on the face. Facial recognition has made humans various emotional vulnerabilities. Artificial intelligence shows their lack of transparency making public inquiry almost impossible.

\section{B. Security}

Security; passwords can be cracked through the various micro facial expressions and they can be retrieved and used against the targeted groups. The software developers also have full authority over the data used to develop the AI, the data can either be leaked or hacked and used against the individuals involved. Human rights and freedom of expression based on the report by the authorities show that they face grave consequences. As stated above the emotion detection technologies are prone to spoofing and hacking, this has expanded the breaching public data and other added data vulnerabilities like erasing of the data and change of data.

\section{The Ethical Issues of Accuracy}

It is most likely that this software may inappropriately misinterpret the situations since there are many aspects in emotion recognition. Greater accuracy and ease in insistent monitoring comes along with concerns that are beyond ethics. Context is everything if you want to read someone's emotions e.g. if a mother cries during birth or after the birth of her child it is likely to be out of happiness or if a footballer Puffs out their chest and exposes their teeth after they score a goal they are probably celebrating the score and not anything violent. This has resulted in us knowingly regulating the signals we send to others, it has impacted the release of lie detection technology. The system's goal is to detect lies in people.

\section{The Dilemma of Discrimination}

Discrimination may occur because of doubtful accuracy in women, children, non-whites, and ethnic minorities (Koshimizu, 2006). AI also gives rise to discrimination since its facial expressions are based on outdated science. The research and study of facial recognition have shown that the technology promotes discrimination since it was produced, implemented, and used in western society. It also promotes the unhealthy effects of racism.

\section{CONCLUSION}

Emotions play a powerful role in people's thinking and behaviors. Emotions act as a compulsion to take any action and can influence daily life decisions. Therefore a larger percentage of human communications is through facial expressions. Gaining an understanding of the contexts of emotions has a set of impacts on society and businesses. Therefore there was a need to develop an emotion detecting algorithm, the algorithm is used to detect certain emotions depicted by humans and the intentions of the various emotions. This is widespread in corporations and governmental institutions like the criminal justice system. However, the computer vision in detecting human reactions poses ethical dilemmas to the general public and makes the public vulnerable to manipulations.

\section{REFERENCES}

1. Amjad, K. M. (2020). A Technique and Architectural Design for Criminal Detection based on Lombroso Theory Using Deep Learning. LGURJCSIT, 4(3), 47-63.

2. Anusha, P. P. (2020). FACIAL DETECTION IMPLEMENTATION USING PRINCIPAL COMPONENT ANALYSIS (PCA). Journal of Critical Reviews, 7(10), 1863-1872.

3. Dzedzickis, A. K. (2020). Human emotion recognition: Review of sensors and methods. Sensors, 20(3), 592.

4. Dzedzickis, A. K. (2020). Human emotion recognition: Review of sensors and methods. . Sensors, 20(3), 592.

5. Koshimizu, T. T. (2006, October ). Factors on the sense of privacy in video surveillance. In Proceedings of the 3rd ACM workshop on Continuous archival and retrieval of personal experiences, pp. 35-44.

6. McStay, A. (2020). Emotional AI and EdTech: serving the public good? Learning, Media and Technology, 45(3), 270-283.

7. Mehta, D. S. (2018). Facial emotion recognition: A survey and real-world user experiences in mixed reality. Sensors, 18(2), 416.

\section{AUTHOR'S PROFILE}

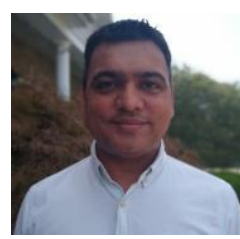

Ravindra Kumar, is a technologist in the healthcare IT, population health management, clinical software applications product management, and Implementation space. Ravindra Kumar is interested in social use of Artificial Intelligence and spending his time in researching how to find ways using AI to save human life and make human life better and easy. He has over fifteen years of proven experience in leading and making global teams spanning various functions like Technical, Solutions Architecture, Technical Alliances, Development, and Quality. He is passionate about value-based care and is actively involved in designing and implementing new approaches for data-driven initiatives for population health management for Medicare Advantage and ACO markets. In Past, Ravindra was involved in end to end implementation of Medicare Advantage plans, and Population health IT systems. He has good knowledge of care management, Utilization Management and other applications for value based care. He has a great interest in healthcare data analytics and is pretty comfortable in using Tableau, SQL, PowerBI for analysis of various healthcare data (Claims, eligibility, provider data, etc), including EMRs, HL7, ADT and FHIR records. He also loves to train people in quality practices, including RPA(Robotic Process Automation), Test Automation, and Agile engineering.

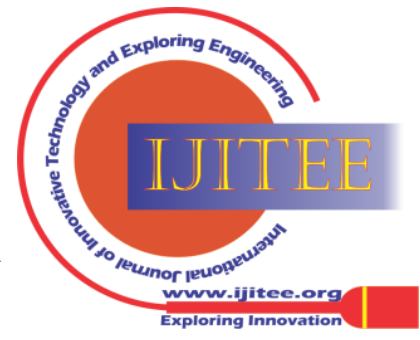

